\title{
Ultrahigh-Spectral-Efficiency WDM/SDM Transmission Using PDM-1024-QAM Probabilistic Shaping With Adaptive Rate
}

Hu, Hao; Yankov, Metodi Plamenov; Da Ros, Francesco; Amma, Yoshimichi; Sasaki, Yusuke; Mizuno, Takayuki; Miyamoto, Yutaka; Galili, Michael; Forchhammer, Søren; Oxenløwe, Leif Katsuo Total number of authors:

11

Published in:

Journal of Lightwave Technology

Link to article, DOI:

10.1109/JLT.2017.2787340

Publication date.

2018

Document Version

Peer reviewed version

Link back to DTU Orbit

Citation $(A P A)$ :

Hu, H., Yankov, M. P., Da Ros, F., Amma, Y., Sasaki, Y., Mizuno, T., Miyamoto, Y., Galili, M., Forchhammer, S., Oxenløwe, L. K., \& Morioka, T. (2018). Ultrahigh-Spectral-Efficiency WDM/SDM Transmission Using PDM-1024QAM Probabilistic Shaping With Adaptive Rate. Journal of Lightwave Technology, 36(6), 1304-1308. https://doi.org/10.1109/JLT.2017.2787340

\section{General rights}

Copyright and moral rights for the publications made accessible in the public portal are retained by the authors and/or other copyright owners and it is a condition of accessing publications that users recognise and abide by the legal requirements associated with these rights.

- Users may download and print one copy of any publication from the public portal for the purpose of private study or research.

- You may not further distribute the material or use it for any profit-making activity or commercial gain

- You may freely distribute the URL identifying the publication in the public portal 


\title{
Ultra-High-Spectral-Efficiency WDM/SDM Transmission using PDM-1024-QAM Probabilistic Shaping with Adaptive Rate
}

\author{
Hao Hu, Metodi Plamenov Yankov, Francesco Da Ros, Yoshimichi Amma, Yusuke Sasaki, \\ Takayuki Mizuno, Yutaka Miyamoto, Michael Galili, Søren Forchhammer, Leif K. Oxenløwe, and \\ Toshio Morioka
}

(Invited Paper)

\begin{abstract}
We demonstrate wavelength-division-multiplexed (WDM) and space-division-multiplexed (SDM) transmission of probabilistically shaped polarization-division-multiplexed (PDM) 1024-state quadrature amplitude modulation (QAM) channels over a 9.7-km single-mode 30-core fiber, achieving aggregated spectral efficiency of $297.82 \mathrm{bit} / \mathrm{s} / \mathrm{Hz}$ on a 12.5-GHz grid and 7.01Tbit/s spatial-super-channel on a 25-GHz grid without multipleinput multiple-output (MIMO) processing. Actual soft-decision forward error correction (SD-FEC) decoding was employed to obtain error-free performance, and adaptive rates and spectral efficiencies for individual WDM/SDM channels have been applied according to their channel conditions, by adjusting the SD-FEC overhead without changing the modulation format. Probabilistically shaped PDM-1024-QAM has been used to further increase the aggregated achievable rate due to the added performance improvement through shaping gain.
\end{abstract}

Index Terms - Optical fiber communication, wavelength division multiplexing (WDM), space division multiplexing (SDM), spectral efficiency, adaptive rate, probabilistic shaping, forward error correction (FEC), 1024-state quadrature amplitude modulation (1024-QAM).

\section{INTRODUCTION}

$\mathrm{T}^{\circ}$ meet the ever-increasing demand for large-capacity optical fiber transmission, space-division multiplexing (SDM) based on single-mode multi-core fibers (SM-MCFs) or few-mode multi-core fibers (FM-MCFs) has been proposed to significantly increase the aggregated spectral efficiency and transmission capacity [1-3]. Compared to the FM-MCF

Manuscript received. This work was partly supported by the Danish National Research Foundation (DNRF) under the Centre of Excellence SPOC (Centre for Silicon Photonics for Optical Communications, ref DNRF123) and partly supported by the MIC, Japan and EC Horizon 2020 under the EU-Japan project on "Scalable And Flexible optical Architecture for Reconfigurable Infrastructure (SAFARI)".

Hao Hu, Metodi Plamenov Yankov, Francesco Da Ros, Michael Galili, Søren Forchhammer, Leif K. Oxenløwe, and Toshio Morioka are with the DTU Fotonik, Department of Photonics Engineering, Technical University of Denmark, DK-2800, Kgs. Lyngby, Denmark (email: huhao@fotonik.dtu.dk).

Yoshimichi Amma and Yusuke Sasaki are with Advanced Technology Laboratory, Fujikura Ltd., Sakura, Chiba, 285-8550, Japan.

Takayuki Mizuno and Yutaka Miyamoto are with the NTT Network Innovation Laboratories, NTT Corporation, Hikarinooka, Yokosuka, Kanagawa, 239-0847, Japan. system, the transmission system using SM-MCF with uncoupled cores has the advantage of being free from mode dependent loss and modal differential group delay, thus removing the need for computation-intensive multiple-input multiple-output (MIMO) processing and lowering both power consumption and latency. In order to boost the transmission capacity, dense SDM based on high-count SM-MCFs over 30 cores have been demonstrated recently [4-5].

In order to further increase data rate in the high-count SMMCF transmission system, more advanced modulation formats with higher spectral efficiency (SE), such as higher-order quadrature amplitude modulation (QAM), will be needed. While 1024-QAM and 2048-QAM have been used for singlechannel SM-MCF transmission with a symbol rate of only $\sim 3$ Gbaud [6-7], the most advanced modulation format used for the wavelength-division multiplexing (WDM) /SDM transmission system so far is polarization-division-multiplexed (PDM)-64-QAM [2, 8]. To achieve higher-order QAM (such as 1024-QAM) with higher symbol rates (>10 Gbaud) over multiple WDM channels, there are several main challenges such as limitations of current transceivers, achievable optical signal-to-noise ratio (OSNR) after the transmission and cost of narrow-linewidth lasers in the system. Recently, probabilistic shaping has attracted attentions in optical communications, not only for achieving shaping gain but also for the capability of adapting their rate to channel conditions [9-11].

A large-capacity WDM/SDM transmission system typically consists of thousands or tens of thousands of parallel channels in both wavelength and space $[1-5,8]$ domains, which usually do not exhibit the same transmission performance due to unequal gain of optical amplifier over wavelengths, loss variation of spatial channels and power variation among all the channels. However, the overall transmission performance is usually limited by the worst channels, in order to achieve error-free performance for all the transmitted channels. By using adaptive rates for individual channels according to the channel conditions, data rates for all the channels can be maximized, thus increasing the aggregated transmission rate and spectral efficiency [12]. 


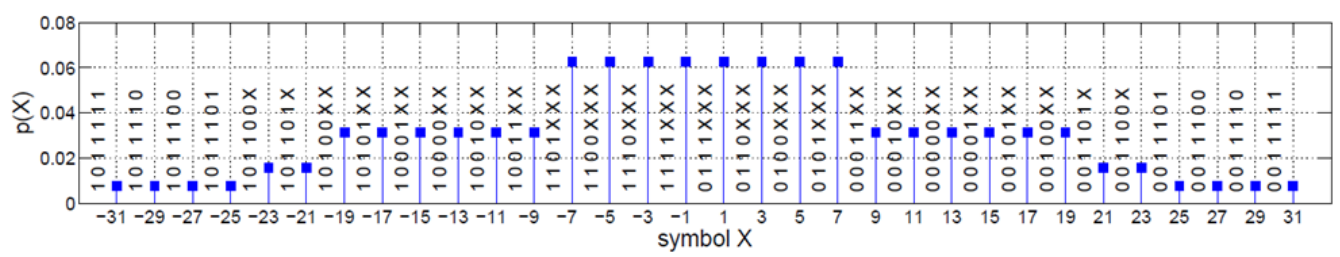

Fig. 1. The symbols have probabilities of $p\left(x_{i}\right)=2^{-l_{i}}$, where $l$ is an integer. 32 symbols in each I and Q, resulting in 1024-QAM.

In this paper, we demonstrate the first probabilistic shaping (PS) for WDM/SDM transmission with ultra-high aggregated spectral efficiency. We employ the advanced modulation format of PS-PDM-1024-QAM and full soft-decision forward error correction (SD-FEC) decoding with adaptive information rates for different spectral and spatial channels to maximize data rates with error-free performances. We achieve recordhigh spectral efficiency of up to $297.8 \mathrm{bit} / \mathrm{s} / \mathrm{Hz}$ for a SM-MCF transmission system. The inter-core crosstalk of the singlemode 30-core fiber used in the experiment is low enough, thus allowing for MIMO-free transmission.

The paper is organized as follows. We first describe the coding and decoding of 1024-QAM with probabilistic shaping and SD-FEC in Section II. Then, we present the experimental setup of the WDM/SDM transmission of PS-PDM-1024-QAM channels in section III. Experimental results after the WDM/SDM transmission are shown in Section IV. Finally, we conclude the paper in Section V.

\section{ProbabILISTIC SHAPING AND SD-FEC}

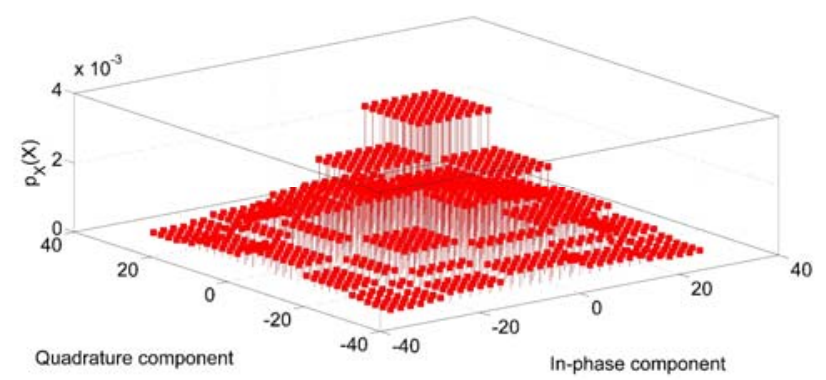

Fig. 2. 2D histogram of probability mass function (PMF) for 1024-QAM.

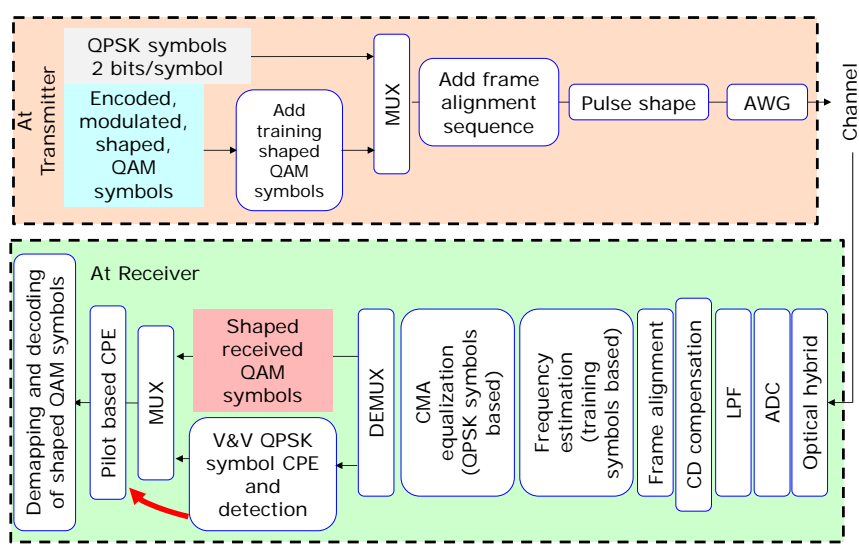

Fig. 3. Diagram of the transmitter and the receiver with hybrid shaped QAM/QPSK based digital signal processing.
Probabilistic shaping is performed as in [9]. The user data are encoded by a turbo code with convolutional constituent encoders, and then many-to-one bit-to-symbol mapped. The symbol probabilities of $p\left(x_{i}\right)=2^{-l_{i}}$ ( $l$ is an integer) is used in the experiment, in order to achieve the optimized probability mass function (PMF) of the QAM symbols shown in Fig. 1. With many-to-one labeling, in contrast to standard QAM with unique Gray mapping, multiple bit strings are mapped to the same constellation symbol, making it appear more often on average (Fig.2). This results in increased length of the symbol label, e.g. 14 bits/symbol for the shaped 1024-QAM constellation considered here, and the resulting ambiguities are resolved by the decoder. The adaptive rate in this scheme is achieved by properly puncturing the turbo code to the desired SD-FEC overhead and data rates of $\xi=5,5.5$ and 6 information bits/QAM symbol are used. Due to the rather large constellation size (1024-QAM) and the fact that the total bit-rate is increased for many-to-one mapping w.r.t. standard Gray mapping, the required overhead is $180 \%, 154.5 \%$ and $133.3 \%$ for the three data rates, respectively. The PS QAM symbols are then uniformly interleaved with $10 \%$ QPSK symbols which carry $\xi_{Q P S K}=2$ bits/QAM symbol, resulting in a hybrid modulation, as shown in Fig. 3. Finally, a frame alignment sequence (FAS) is added to the beginning of the sequence, pulse shaping is performed (square root raised cosine, roll-off 0.01 ), and the waveform is sent to the arbitrary waveform generator (AWG).

At the receiver, the FAS is found, which allows for identifying the QPSK symbol positions. The adaptive constant modulus algorithm is only performed on the QPSK symbols. The equalization taps are found and then interpolated to equalize the remaining QAM symbol positions [13]. Using the QPSK symbols, Viterbi\&Viterbi (V\&V) coarse frequency offset and phase noise correction is performed, which allows for demodulation of the QPSK data (the achievable receiver OSNR is well above the required OSNR for QPSK to reach its error-free decoding threshold). Once demodulated, the QPSK symbols are used as pilots for fine phase noise compensation as in [9].

Iterative demapping and decoding is performed with 10 iterations between the turbo code's constituent decoders for each of 5 demapping iterations. As mentioned in [9], iterative demapping, while beneficial for the system overall, is not a requirement for achieving shaping gain for the studied data rates and mapping functions. More than 300 blocks of 6600 (6000 QAM + 600 QPSK) symbols are transmitted and decoded in each case, making the number of decoded information bits $>10^{7}$ in all cases and thereby BER values above $10^{-5}$ reliable. Consequently, when no errors are measured, the BER is reliably below $10^{-5}$. An outer HD-FEC is then assumed with overhead $0.8 \%$ and decoding threshold 
of $5 \times 10^{-5}$ [14], which can correct the remaining errors and bring the BER below $10^{-15}$. When the BER $\leq 10^{-5}$ is measured, it is considered reliably error-free after the HD-FEC decoding.

The system performance in this paper is evaluated based on the mutual information between input and output sequences, as well as achieved net data rate with error-free $\left(\mathrm{BER} \leq 10^{-5}\right)$ performance. The MI describes an achievable information rate (AIR) with ideal FEC and poses an upper bound on the achieved net data rate with tractable, finite-length error correction. The MI is estimated for the hybrid sequence in [bits/QAM symbol] and thus includes the effect of the QPSK symbols. The AIR is given by

$$
A I R=\eta \cdot \frac{1}{1.1}\left(\mathcal{J}\left(X_{Q A M} ; Y_{Q A M}\right)+0.1 \cdot \mathcal{J}\left(X_{Q P S K} ; Y_{Q P S K}\right)\right),
$$

where $\eta$ is the bandwidth occupancy, $X_{Q A M}$ and $Y_{Q A M}$ are the input and output PS-1024-QAM symbols respectively, $X_{Q P S K}$ and $Y_{Q P S K}$ are the input and output symbols (after V\&V phase noise correction) at the QPSK positions and $\mathcal{J}(\because ;)$ is the MI functional. A typical received QPSK constellation (defined by the symbols $Y_{Q P S K}$ ) is given in Fig. 4. The estimated errorvector magnitude $(E V M)$ of the symbols is $\approx 11.5 \%$, which results in estimated $B E R_{Q P S K}^{e s t} \approx 2 \mathrm{e}-18$. In all our experiments, the measured $\quad B E R_{Q P S K}^{\text {meas }}=0 . \quad$ Correspondingly, $\mathcal{J}\left(X_{Q P S K} ; Y_{Q P S K}\right)$ was estimated to coincide with the maximum data rate, obtainable by QPSK constellations of $\mathcal{J}\left(X_{Q P S K} ; Y_{Q P S K}\right)=\xi_{Q P S K}=\log _{2} 4=2$ bits/symbol. The MI $\mathcal{J}\left(X_{Q A M} ; Y_{Q A M}\right)$ is estimated with the auxiliary channel and mismatched decoding principle [15]. In particular, the symbols $X_{Q A M}$ and $Y_{Q A M}$ are assumed to be the input and output, respectively, of a standard Wiener phase noise channel [16]. The MI is estimated with the phase noise compensation algorithm from [16].

Assuming the above mentioned HD-FEC, the resulting net $\mathrm{SE}$ is calculated as

$$
S E=\eta \cdot \frac{\left(\xi+0.1 \cdot \xi_{Q P S K}\right)}{1.1} \cdot \frac{1}{1+0.008}
$$

The SE is achieved when the BER after SD is found to be $\leq 10^{-5}$ (see explanation above). Consequently, the difference between the SE and the AIR describes the penalty from the imperfect coding.

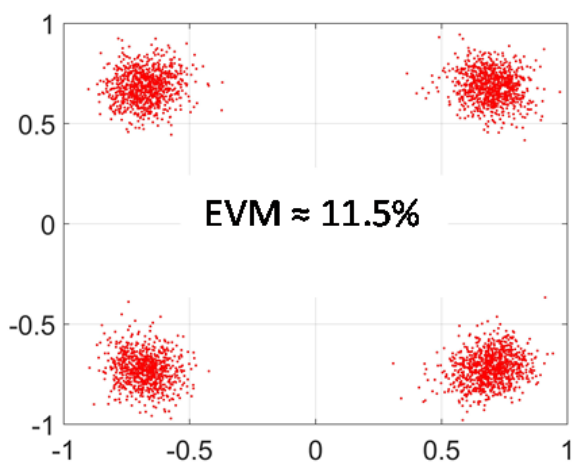

Fig. 4. Received constellation for the QPSK symbols. EVM is approximately $11.5 \%$, corresponding to estimated BER of $\sim 2 \times 10^{-18}$, and measured BER $=0$.

\section{EXPERIMENTAL SETUP}

Figure 5 (a) shows the experimental setup for the WDM/SDM transmission of PS PDM-1024-QAM channels. Eight external cavity lasers (ECLs) centered around $193.4 \mathrm{THz}$ and with a linewidth of $10 \mathrm{kHz}$ are divided into odd and even channels, and then independently modulated with two I/Q modulators driven by a 4-channel arbitrary waveform generator (AWG) with a sampling rate of $64 \mathrm{GSa} / \mathrm{s}$ to produce PS 1024-QAM WDM channels. In the experiment, two symbol rates and frequency grids are used, 12 Gbaud on a $12.5 \mathrm{GHz}$ grid or $24.5 \mathrm{Gbaud}$ on a $25 \mathrm{GHz}$ grid. The modulated odd and even channels are combined by a $3-\mathrm{dB}$ coupler with an additional delay in one path for further decorrelation between (a)

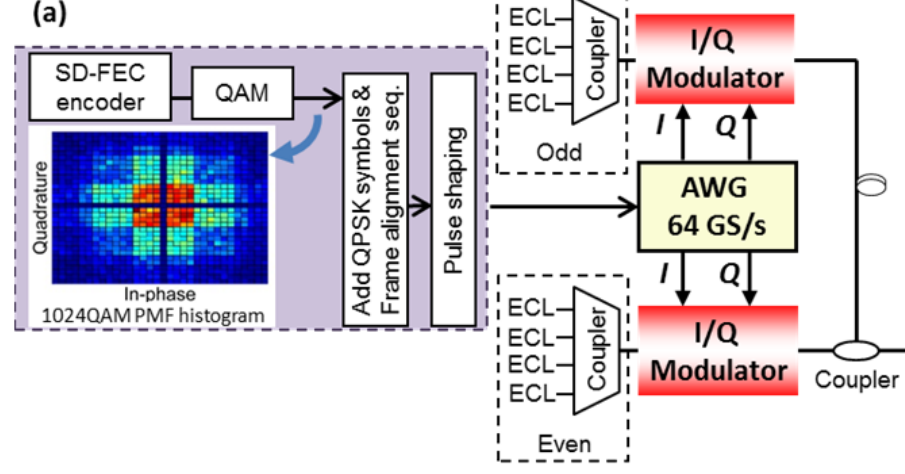

(b)
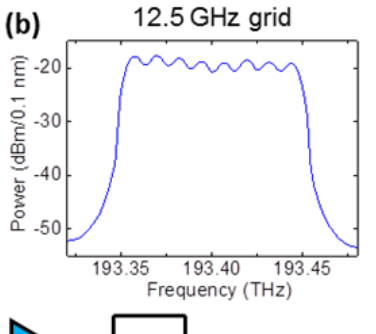

(d)

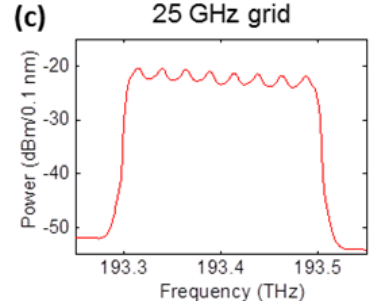

(e)
EDFA Pol. Mux

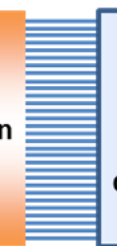

Splitting

and

decorrelation

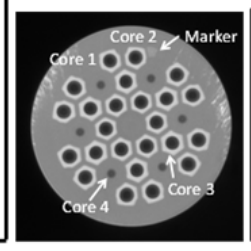

$80 \mathrm{GS} / \mathrm{s}$

$32 \mathrm{GHz}$

EDFA

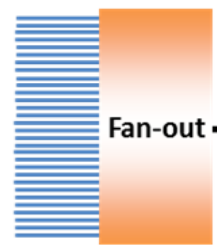

$9.6 \mathrm{~km}$ 30-core fiber

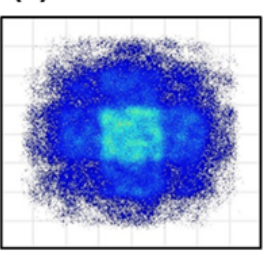

Fig. 5. (a) Schematic of the experimental setup for the WDM/SDM transmission of PS PDM-1024-QAM channels over a 30-core fiber, including external cavity lasers (ECLs), an arbitrary waveform generator (AWG), erbium-doped fiber amplifiers (EDFAs) and an optical bandpass filter (OBF); (b) and (c) optical spectra of the WDM channels on a $12.5 \mathrm{GHz}$ grid and a $25 \mathrm{GHz}$ grid after the 30-core fiber transmission; (d) cross-sectional view of the 30 -core fiber; (e) constellation of the probabilistic shaped 1024-QAM with a SNR of $20.3 \mathrm{~dB}$ after the transmission before carrier phase recovery. 
neighboring channels, amplified by an EDFA and then passed through a delay-and-add polarization emulator to generate PDM signals. The generated WDM channels with a $12.5 \mathrm{GHz}$ grid or a $25 \mathrm{GHz}$ grid are shown in Fig. 5 (b) and (c), respectively. Furthermore, 30 de-correlated SDM channels are generated using splitters, amplifiers and delays (at least $2.5 \mathrm{~ns}$ between SDM channels), which are then launched into a 9.6$\mathrm{km}$ heterogeneous single-mode 30 -core fiber through a $3 \mathrm{D}$ waveguide based fan-in device. The launched power for each core varies from 4 to $8 \mathrm{dBm}$, since the fan-in/fan-out devices have loss-variations in between $5 \mathrm{~dB}$ and $8 \mathrm{~dB}$.

The heterogeneous single-mode 30 -core fiber used in the experiment has four different types of cores (Fig. 5 (d)), which introduce phase mismatch between neighboring cores, resulting in a high-density core arrangement with low crosstalk of $<-50 \mathrm{~dB}$ after a $9.6 \mathrm{~km}$ transmission [17]. The low inter-core crosstalk enables such a high data rate transmission without MIMO processing. The 30 cores are arranged within the limited cladding diameter of $228 \mu \mathrm{m}$, with an average $\mathrm{A}_{\mathrm{eff}}$ of approximately $80 \mu^{2}$.

At the output of the 30 -core fiber, 30 SDM channels are demultiplexed using another 3D-waveguide based fan-out device. The demultiplexed spatial channels are amplified, and one WDM channel is filtered out at a time. Each selected WDM channel is detected by a polarization-diversity coherent receiver followed by a digital sampling oscilloscope (DSO, $80 \mathrm{GSa} / \mathrm{s}, 33 \mathrm{GHz}$ ) and offline digital signal processing, as described in Section II and Fig. 3.

\section{EXPERIMENTAL RESULTS}

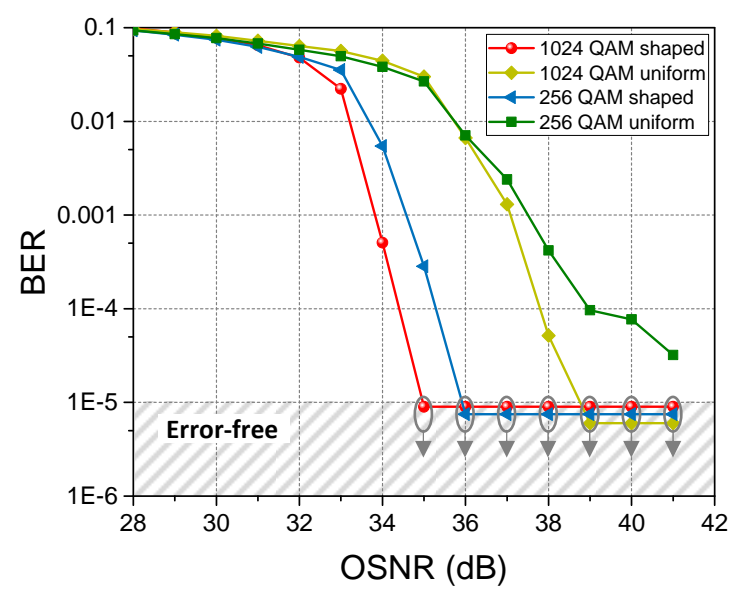

Fig. 6. BER performances of uniform QAM signal and probabilistic shaped QAM signal after the WDM/SDM transmission, for the cases of both PDM256-QAM and PDM-1024-QAM at 24.5 Gbaud with an input data rate of 5 bits/QAM symbol.

We first evaluated the transmission performances of uniform QAM signal and probabilistic shaped QAM signal for the central WDM channel. Both PDM-256-QAM and PDM-1024QAM at 24.5 Gbaud with an input data rate of 5 bits/QAM symbol are studied. As previously demonstrated in [9], the pilot-based DSP employed in this work results in modulation format-independent implementation penalty. As shown in Fig.6, the performance of PS PDM-1024-QAM is $1 \mathrm{~dB}$ better than the PS PDM-256-QAM and $4 \mathrm{~dB}$ better than the uniform
PDM-1024-QAM at a BER of $10^{-5}$, and the uniform PDM256-QAM cannot achieve BER below $10^{-5}$ with the available OSNR. This result indicates that the transmission reach can be increased by using PS PDM-1024-QAM compared to other modulation formats since it requires the lowest OSNR for error-free performance. It is noted that the operating point is at high OSNR, where the transceiver performance saturates to its maximum, limited by the implementation penalties. At such regions, the electrical SNR improves very slowly with OSNR, which is the reason for the large OSNR shaping gain. The estimated effective SNR at the error-free OSNR point of 35 $\mathrm{dB}$ was $\mathrm{SNR}_{\mathrm{eff}} \approx 16.8 \mathrm{~dB}$, for which the Shannon capacity is $\eta \cdot \log _{2}\left(1+\mathrm{SNR}_{\mathrm{eff}}\right)=5.49 \mathrm{bits} / \mathrm{s} / \mathrm{Hz}$. The penalties to the Shannon limit are attributed mainly to the imperfect phase noise compensation and the fact that the spectral efficiency is lower due to the $10 \%$ QPSK hybrid.

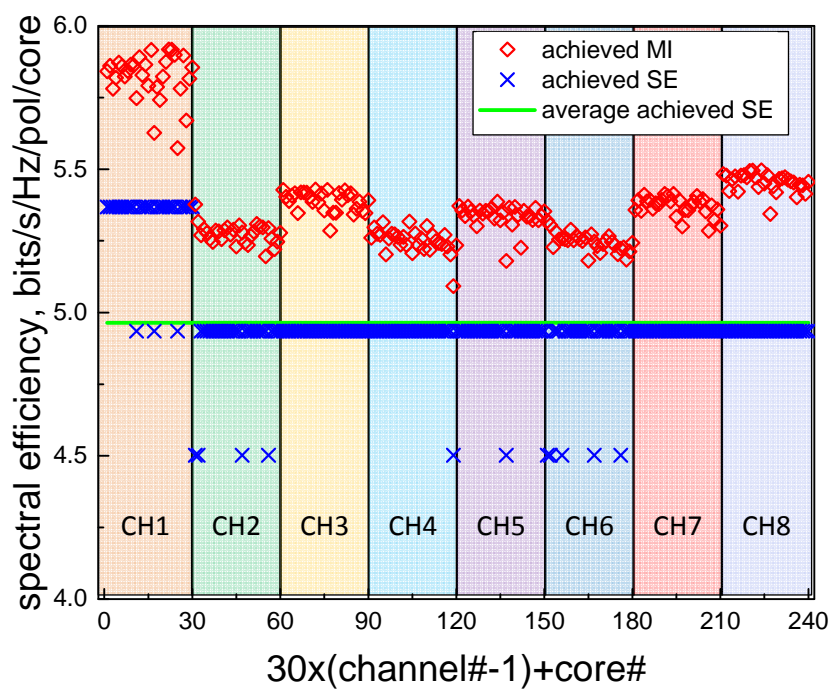

(a)

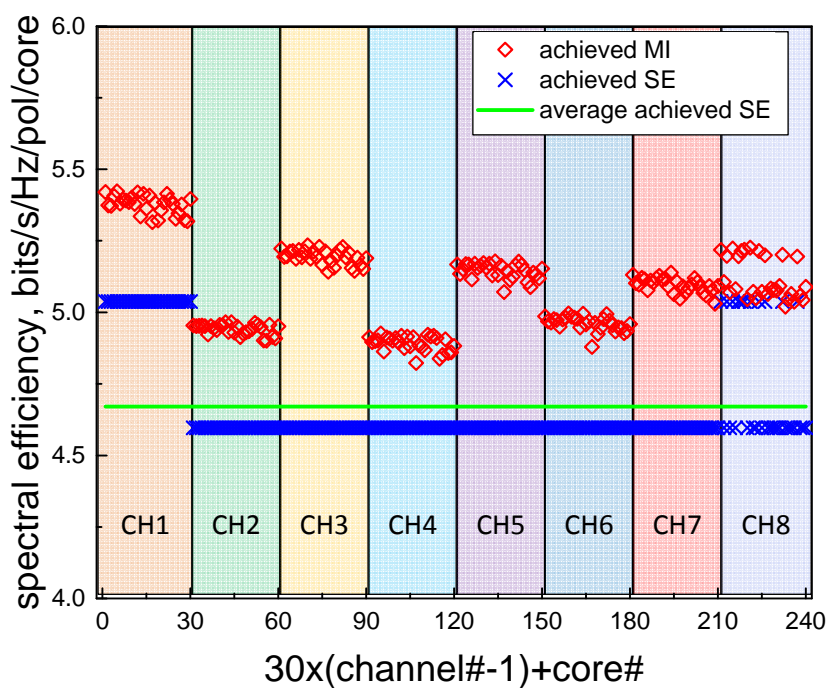

(b)

Fig. 7. Achieved mutual information (MI, bits/QAM symbol) and achieved spectral efficiency (SE, bits/s/Hz/pol/core) using the employed non-ideal SDFEC decoding after the 30-core transmission of all the WDM and SDM channels for the cases of 12 Gbaud PS PDM-1024-QAM signal on a $12.5 \mathrm{GHz}$ grid (a) and 24.5 Gbaud PS PDM-1024-QAM signal on a $25 \mathrm{GHz}$ grid (b). Channel\# corresponds to the WDM channels (from 1 to 8 ) and core\# corresponds to the cores of the 30-core fiber (from 1 to 30 ). 


\begin{tabular}{|c|c|c|c|c|c|c|c|c|c|}
\hline & \multirow{2}{*}{$\begin{array}{c}\text { Outer } \\
\text { HD-FEC } \\
\text { overhead }\end{array}$} & \multirow{2}{*}{$\begin{array}{l}\text { QPSK } \\
\text { hybrid }\end{array}$} & \multicolumn{3}{|c|}{$\begin{array}{c}\text { Tested input data rate } \xi \\
\text { (shaping) [bits/QAM symbol] }\end{array}$} & \multirow{2}{*}{$\begin{array}{c}\text { Achieved } \\
\text { average SE } \\
\text { [bit/s/Hz/pol/ } \\
\text { core] }\end{array}$} & \multirow{2}{*}{$\begin{array}{c}\text { Achieved } \\
\text { average AIR } \\
\text { (hybrid) } \\
\text { [bits/symbol] }\end{array}$} & \multirow{2}{*}{$\begin{array}{c}\text { Achieved } \\
\text { aggregated SE } \\
{[\mathrm{bit} / \mathrm{s} / \mathrm{Hz}]}\end{array}$} & \multirow{2}{*}{$\begin{array}{l}\text { Achieved } \\
\text { aggregated data } \\
\text { rate per frequency } \\
\text { channel [Tbps] }\end{array}$} \\
\hline & & & \multicolumn{3}{|c|}{$\begin{array}{l}\text { Tested input net SE } \\
{[\mathrm{bit} / \mathrm{s} / \mathrm{Hz} / \mathrm{pol} / \text { core] }}\end{array}$} & & & & \\
\hline \multirow{2}{*}{$\begin{array}{c}12 \text { Gbaud@ } \\
12.5 \mathrm{GHz}\end{array}$} & \multirow{4}{*}{$0.8 \%$} & \multirow{4}{*}{$10 \%$} & 5 & 5.5 & 6 & & & & \\
\hline & & & 4.50 & 4.94 & 5.27 & 4.96 & 5.39 & 291.82 & 3.12 \\
\hline \multirow{2}{*}{$\begin{array}{l}24.5 \mathrm{Gbaud} \\
\text { @ } 25 \mathrm{GHz}\end{array}$} & & & 5 & 5.5 & 6 & \multirow{2}{*}{4.67} & \multirow{2}{*}{5.09} & \multirow{2}{*}{280.27} & \multirow{2}{*}{7.01} \\
\hline & & & 4.60 & 5.04 & 5.48 & & & & \\
\hline
\end{tabular}

Table 1. Summary of the main results.

Fig. 7 shows the AIR in bits/QAM symbol and the achieved spectral efficiency (SE, bits/s/Hz/polarization/core) with errorfree performance after the 30-core fiber transmission for all the WDM and SDM channels in the cases of 12 Gbaud PS PDM-1024-QAM signal on a $12.5 \mathrm{GHz}$ grid and 24.5 Gbaud PS PDM-1024-QAM signal on a $25 \mathrm{GHz}$ grid. The MI gives an upper bound on the achievable SE by using ideal SD-FEC decoding. We also employed actual SD-FEC decoding to achieve error-free performance after the transmission, and the experimentally achieved SE based on our non-ideal codes is also shown.

Due to the OSNR variations between the WDM channels and loss variations in the different cores of the 30-core fiber, transmission performances of the WDM/SDM channels are not the same. Since the channel $1(\mathrm{CH} 1)$ is at the edge of the WDM channels, it has less inter-channel crosstalk and less nonlinear distortion compared to the channels in the middle. In addition, $\mathrm{CH} 1$ has slightly higher power than the other WDM channels due to gain tilting of the EDFA. Therefore, $\mathrm{CH} 1$ has better performance than the other channels. Three different input data rates $(5,5.5$ and 6 bits/QAM symbol) were tested for each individual channel, and maximum MIs and SEs are achieved with error-free performance according to the channel conditions, thus enabling adaptive rates and SEs for different WDM/SDM channels without changing the modulation format. Table 1 summarizes the main results of the experiment. For the 12-Gbaud PS PDM-1024-QAM signal on a $12.5 \mathrm{GHz}$ grid, the achieved average AIR is 5.39 bits/QAM symbol and the achieved average SE with the employed FEC decoding is $4.96 \mathrm{bit} / \mathrm{s} / \mathrm{Hz} / \mathrm{pol} / \mathrm{core}$, resulting in an aggregated spectral efficiency of $297.82 \mathrm{bit} / \mathrm{s} / \mathrm{Hz}$ and an aggregated net data rate per frequency channel of $3.72 \mathrm{Tbit} / \mathrm{s}$. For the 24.5-Gbaud PS PDM-1024-QAM signal on a $25 \mathrm{GHz}$ grid, the achieved average MI is 5.09 bits/QAM symbol and the achieved average SE with employed FEC decoding is 4.76 $\mathrm{bit} / \mathrm{s} / \mathrm{Hz} / \mathrm{pol} / \mathrm{core}$, resulting in an aggregated spectral efficiency of $280.27 \mathrm{bit} / \mathrm{s} / \mathrm{Hz}$ and an aggregated net data rate per frequency channel of $7.01 \mathrm{Tbit} / \mathrm{s}$.

\section{CONCLUSION}

In order to maximize overall capacity and spectral efficiency, we have demonstrated adaptive rates and spectral efficiencies for individual WDM/SDM channels according to their channel conditions, by adjusting the SD-FEC overhead without changing the modulation format. PS PDM-1024-QAM has been used for the WDM/SDM transmission over a 9.6-km 30core fiber, which exhibits lowest OSNR requirement for the error-free performance compared to the other tested modulation formats, including PS PDM-256-QAM, uniform PDM-1024-QAM and uniform PDM-256-QAM. Actual SDFEC decoding was employed to obtain error-free performance, and owing to the shaping gain we achieved aggregated net data rate per frequency channel of $7.01 \mathrm{Tbit} / \mathrm{s}$ on a $25 \mathrm{GHz}$ grid and record-high aggregate spectral efficiency of up to $297.82 \mathrm{bit} / \mathrm{s} / \mathrm{Hz}$ for SM-MCF transmission systems on a 12.5 $\mathrm{GHz}$ grid. In addition, the inter-core crosstalk of the 30-core fiber is low enough so that MIMO processing is avoided.

\section{REFERENCES}

[1] H. Takara et al., "1.01-Pb/s (12 SDM/222 WDM/456 Gb/s) Crosstalkmanaged Transmission with $91.4-\mathrm{b} / \mathrm{s} / \mathrm{Hz}$ Aggregate Spectral Efficiency," Proc. ECOC, Th.3.C.1, (2012).

[2] B. J. Puttnam et al., " $2.15 \mathrm{~Pb} / \mathrm{s}$ transmission using a 22 core homogeneous single-mode multi-core fiber and wideband optical comb," Proc. ECOC, PDP.3.1 (2015)

[3] D. Soma et al., "10.16 Peta-bit/s Dense SDM/WDM transmission over Low-DMD 6-Mode 19-Core Fibre across C+L Band," Proc. ECOC, Th.PDP.A.1 (2017).

[4] T. Mizuno et al., "32-core Dense SDM Unidirectional Transmission of PDM-16QAM Signals Over 1600 km Using Crosstalk-managed Singlemode Heterogeneous Multicore Transmission Line," Proc.OFC, Th5C.3 (2016).

[5] H. Hu, et al., "Single-Source AlGaAs Frequency Comb Transmitter for $661 \mathrm{Tbit} / \mathrm{s}$ Data Transmission in a 30-core Fiber," in Conference on Lasers and Electro-Optics, OSA Technical Digest (Optical Society of America, 2016), postdeadline paper JTh4C.1 (2016).

[6] M. Yoshida et al., "1024 QAM, 7-core (60 Gbit/s x 7) fiber transmission over $55 \mathrm{~km}$ with an aggregate potential spectral efficiency of 109 bit/s/Hz," Opt. Express, 23(16), p. 20760 (2015).

[7] D. Qian et al., "698.5-Gb/s PDM-2048QAM transmission over $3 \mathrm{~km}$ multicore fiber," Proc. ECOC, Th.1.C.5 (2013).

[8] D. Soma et al., "665 and $947 \mathrm{~b} / \mathrm{s} / \mathrm{Hz}$ Ultra-highly Aggregate-SpectralEfficient SDM/WDM Transmission over 6-Mode 19-Core Fibre Using DP-16QAM/64QAM Signals," Proc. ECOC, Th.3.C.2 (2016).

[9] M. P. Yankov, et. al. "Constellation shaping for WDM systems using 256QAM/1024QAM with probabilistic optimization", J. Lightwave Technol., 34(2), 2016.

[10] S. Chandrasekhar et al., "High-spectral-efficiency transmission of PDM 256-QAM with Parallel Probabilistic Shaping at Record Rate-Reach Trade-offs," Proc. ECOC, Th.3.C.1 (2016).

[11] F. Buchali et al., "Experimental demonstration of capacity increase and rate-adaptation by probabilistically shaped 64-QAM," Proc. ECOC, PDP.3.4 (2015).

[12] H. Hu, M. P. Yankov, F. Da Ros, Y. Amma, Y. Sasaki, T. Mizuno, Y. Miyamoto, M. Galili, S. Forchhammer, L. K. Oxenløwe, and T. Morioka, "Adaptive rates of high-spectral-Efficiency WDM/SDM channels using PDM-1024-QAM probabilistic shaping," Proc. ECOC, Tu.1.D.1 (2017).

[13] M. P. Yankov, E. P. da Silva, F. Da Ros, and D. Zibar, "Experimental analysis of pilot-based equalization for probabilistically shaped WDM systems with 256QAM/1024QAM", Proc. OFC, W2A.48, (2017). 
[14] D. S. Millar, et. al, " Design of a $1 \mathrm{~Tb} / \mathrm{s}$ superchannel coherent receiver", J. Lightwave Technol., 34(6), 2016.

[15] D. M. Arnold, H. Loeliger, P. O. Vontobel, A. Kavčić, and W. Zeng, "Simulation-based computation of information rates for channels with memory," IEEE Trans. Inform. Theory, 52(8), 2006.

[16] M. P. Yankov, T. Fehenberger, L, Barletta, and N. Hanik, "LowComplexity Tracking of Laser and Nonlinear Phase Noise in WDM Optical Fiber Systems", J. Lightwave Technol., 33(23), 2015.

[17] Y. Amma et al., "High-density Multicore Fiber with Heterogeneous Core Arrangement," Proc. OFC, Th4C.4 (2015). 